\title{
An Analytical Approach to the Analysis of Inhomogeneous Pipes under External Pressure
}

\author{
Massimiliano Fraldi and Federico Guarracino \\ Department of Structural Engineering, University of Naples Federico II, via Claudio 21, \\ 80125 Naples, Italy \\ Correspondence should be addressed to Federico Guarracino, fguarrac@unina.it
}

Received 19 February 2012; Revised 6 May 2012; Accepted 7 May 2012

Academic Editor: Ioannis K. Chatjigeorgiou

Copyright (C) 2012 M. Fraldi and F. Guarracino. This is an open access article distributed under the Creative Commons Attribution License, which permits unrestricted use, distribution, and reproduction in any medium, provided the original work is properly cited.

Pipes for deep-water applications possess a diameter-to-thickness ratio in a region where failure is dominated by both instability and plastic collapse. This implies that prior to failure the compressive yield strength of the material must be exceeded, followed by ovalisation and further local yielding. This paper presents an investigation into the mechanics of this specific problem and develops an analytical approach that accounts for the effects of geometrical and material data on the collapse pressure of inhomogeneous rings under external hydrostatic pressure. The analytical expressions have been correlated to numerical and experimental test data, proving their accuracy.

\section{Introduction}

In deep and ultradeep waters the diameter of trunk lines coupled with the hydrostatic pressure tends to lead to failure of the pipeline by external collapse. This failure mode is an instability phenomenon that is governed by the geometry of the pipeline and its material properties. Failure of a pipeline section takes place according to a subtle combination of its properties and to various factors [1-3] and as such the exact limiting collapse pressure can be very difficult to determine. Moreover, on account of fabrication procedures, material properties may vary significantly around the circumference of the pipe.

In the present paper an analytical treatment is developed that can provide adequate prediction of collapse pressure of a pipe section with varying material properties. The proposed approach has its route in the fundamental mechanics of the problem and is capable of blending transitions between elastic collapse for thin-walled structures and plastic collapse for thicker sections. 
Through this understanding of the actual mechanisms involved in the buckling of the pipe it is hoped that further improvements in performance could be gained through optimisation of the pipe manufacturing process, the pipe form, or its mechanical properties.

\section{UOE Forming and Varying Material Properties}

The majority of the pipes used in offshore applications are commonly manufactured by coldforming plates through the UOE process. This means that a steel plate is folded along its edges, formed into a U-shape and then pressed into an O-shape between two semicircular casts. The pipe is successively welded closed and circumferentially expanded to obtain a highly circular shape, see Figure 1.

A large number of experiments have demonstrated that these steps, especially the final expansion, tend to degrade the mechanical properties of the pipe and may occasionally result in a significant variation of the material strength along the circumference of the cross-section. To further enlighten this point, a number of accurate tests on UOE pipes have been recently performed by Tata Steel in accordance with ASTM E9-89a [4]. Samples were taken from the cross-section of a $18^{\prime \prime} \times 27 \mathrm{~mm} \times 65$ pipe, and compression tests were carried out at each of the $0^{\circ}, 90^{\circ}, 180^{\circ}$, and $270^{\circ}$ positions. Generally, the $180^{\circ}$ position was reported to give the lowest result, as shown in Figure 2.

From the graph, in a few cases the difference in compressive strength between the $0^{\circ}$ and $180^{\circ}$ positions was found to be up to $36 \%$ and the difference between $180^{\circ}$ and $90^{\circ} / 270^{\circ}$ positions was found to be up to $14 \%$.

Such variability in material properties may significantly affect the carrying capacity of the pipe and, to the best of authors' knowledge, differently from variations in thickness [5], has never been incorporated in a straight mathematical treatment of the problem, as it will be done in the next section.

\section{An Analytical Approach to Account for the Effects of Material Inhomogeneity}

In order to account for the variability of the material properties around the ring circumference, an analytical treatment that extends the applicability of an already proposed and assessed formulation for uniform rings [6-8] has been developed.

It is worth pointing out that the development focuses on the carrying capacity of a pipe section and that the extension to the case of a long cylindrical shell can be obtained in the Timoshenko fashion [9] by substituting the flexural stiffness of the ring wall, $E t^{3} / 12$, with that pertaining to a shell, that is, $E t^{3} / 12\left(1-v^{2}\right), E$ being Young's modulus of the material, $v$ its Poisson's ratio, and $t$ the wall thickness. Of course, this way of extending the results concerning a cross-section may appear simplistic and to some extent questionable and in fact in the past the problem has received further attention both in the elastic [10] and in the elastic-plastic range [11]. However, given that the Timoshenko solution is firm at the base of most design codes and that the mechanics of the ring buckling is fundamental for a thorough understanding of the phenomenon, focusing on the assessment of the capacity load of rings under external pressure can be considered legitimate, as long as the problem is properly formulated [12]. The present study is in fact conducted along with the point of 


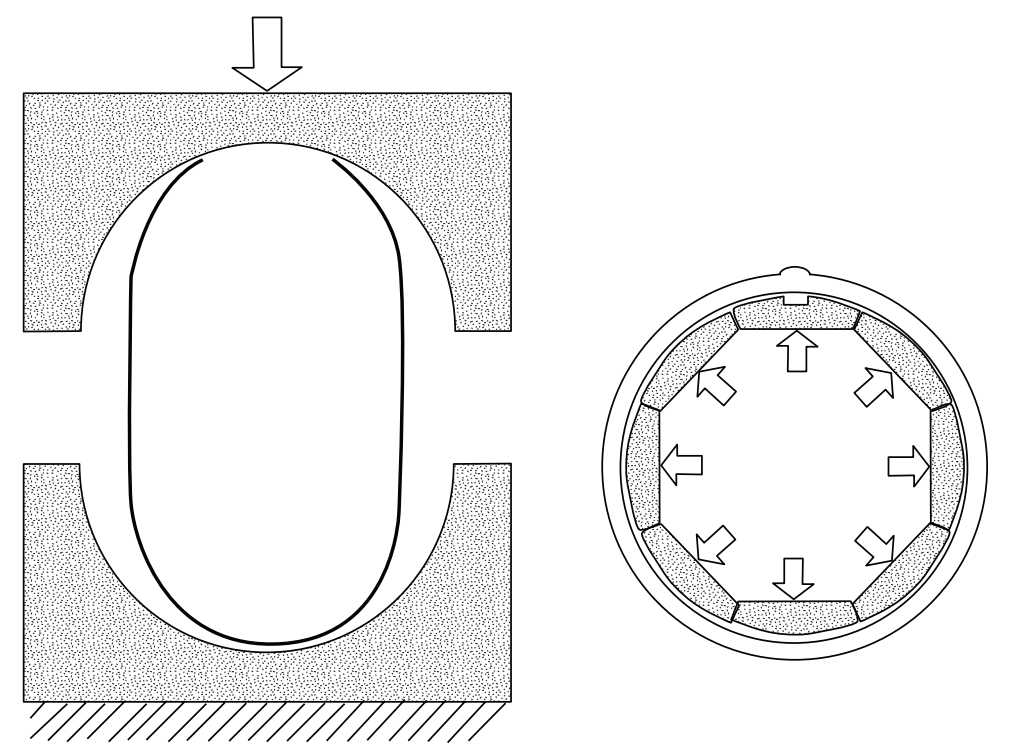

Figure 1: Schematic UOE forming steps.

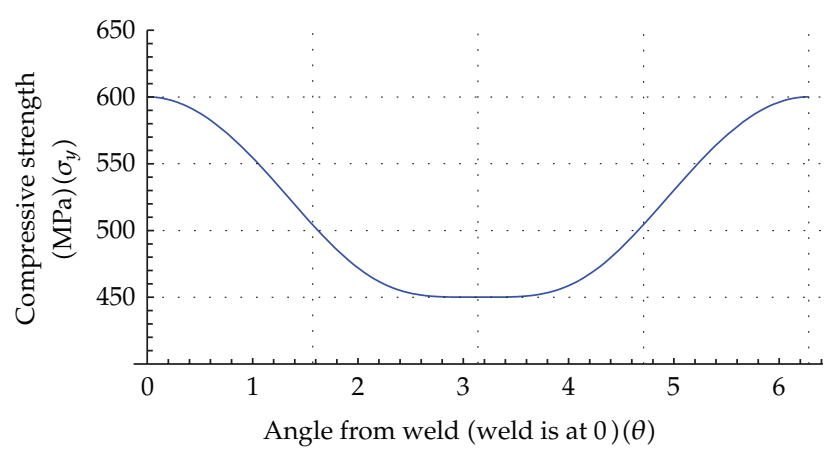

Figure 2: Average strength variation with pipe angular position (Tata Steel, 2010).

view of Charles Massonnet that "in engineering the ability to select the few parameters which really govern the phenomenon is probably the most important quality of the researcher."

With reference to Figure 3 and to a system of polar coordinates,

$$
x=R \sin \theta, \quad y=R \cos \theta,
$$

where $R$ is the mean radius of the ring, the displacement field is expressed in terms of the radial component, $w=w(\theta), p$ being the uniform external pressure. 


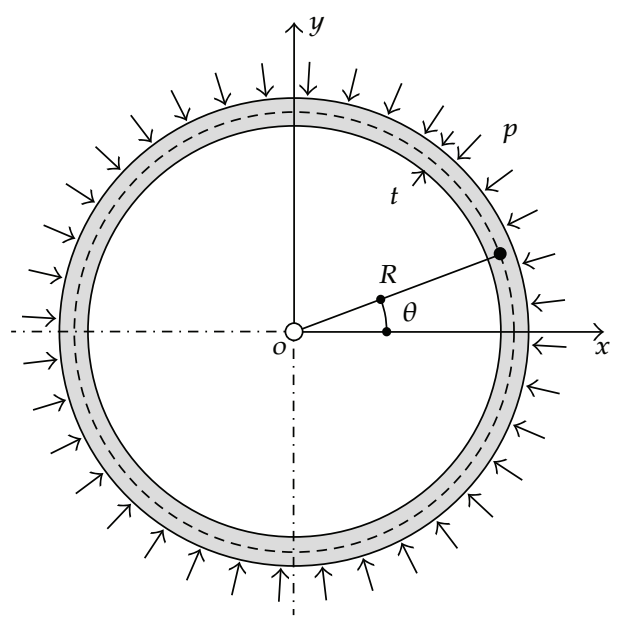

Figure 3: A ring under external pressure.

For the sake of simplicity but without loss of generality, four different material regions, symmetric with respect to the $x$ axis, are defined around the ring circumference, as shown in Figure 4(a). The amplitudes of these regions for $\theta \in[0, \pi]$, see Figure $4(\mathrm{~b})$, are, respectively,

$$
\begin{gathered}
\text { (1) } \theta \in\left[0, \theta_{c}\right], \\
\text { (2) } \theta \in\left[\theta_{c}, \pi-\theta_{c}\right], \\
\text { (3) } \theta \in\left[\pi-\theta_{c}, \pi\right] .
\end{gathered}
$$

Three non-homogeneous second-order linear differential equations, based on the Euler-Bernoulli theory of the elastica, can be written for each of the three sectors with different material properties in which half of the circumference results in being partitioned,

$$
w^{(i) \prime}(\theta)+\left(1+\varphi^{(i)}\right) w^{(i)}(\theta)=-\varphi^{(i)} \omega R \cos n \theta, \quad \omega=w_{0} R^{-1}, n \in N,
$$

with

$$
\varphi^{(i)}=3 p_{e l}^{(i)-1} p=\frac{12}{E_{t}^{(i)} \tau^{3}} p, \quad \tau=t R^{-1}
$$

The superscript $(i)$ indicates the $i$ th material region, $w_{0}$ stands for the amplitude of the initial imperfection, $n$ is the number of waves characterising the out of roundness, $p_{e l}^{(i)}$ represents the Timoshenko critical pressure in the elastic range, and $E_{t}^{(i)}$ is the material Tangent modulus [13]. 


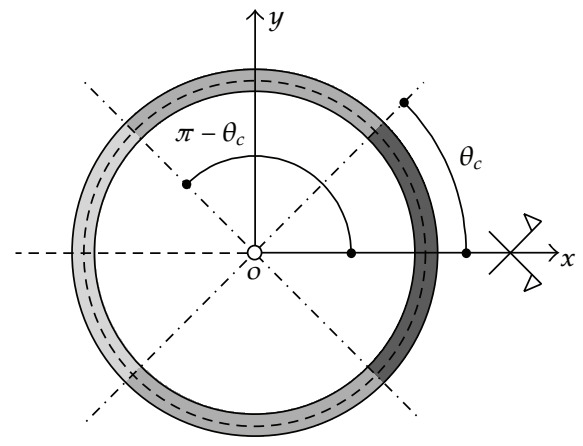

(a)

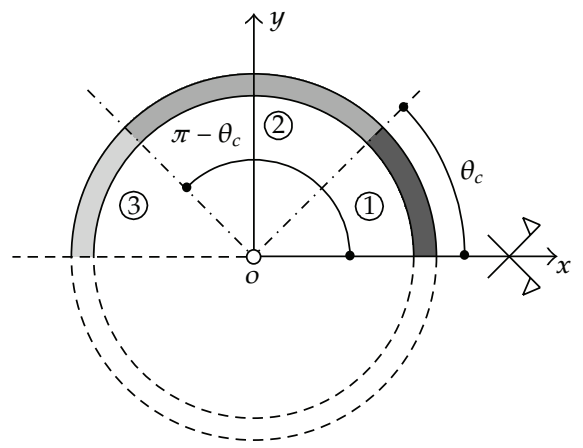

(b)

Figure 4: Nonhomogeneous material regions.

On the basis of experimental evidence it is commonly accepted that the most suitable representation of the stress-strain curves for carbon steels is represented by the RambergOsgood (RO) power law [14],

$$
\varepsilon^{(i)}=\frac{\sigma^{(i)}}{E}+\left(\varepsilon_{y}^{(i)}-\frac{\sigma_{y}^{(i)}}{E}\right)\left(\frac{\sigma^{(i)}}{\sigma_{y}^{(i)}}\right)^{\beta}
$$

where $\beta$ is a dimensionless coefficient, which for most engineering cases can be assumed $\geq 5$, $\sigma_{y}^{(i)}$ is the $i$ th material region yield stress, and $\varepsilon_{y}^{(i)}$ is the corresponding strain. In the following, without loss of generality, Young's modulus, $E$, is assumed to be the same for each material region.

Thus, in such a case the Tangent modulus, $E_{t}^{(i)}$, can be written as

$$
E_{t}^{(i)} \equiv\left(\frac{\partial \varepsilon^{(i)}}{\partial \sigma^{(i)}}\right)^{-1}=E\left(\frac{\sigma_{y}^{(i) \beta}}{\sigma_{y}^{(i) \beta}+\beta\left(E \varepsilon_{y}^{(i)}-\sigma_{y}^{(i)}\right) \sigma^{\beta-1}}\right) .
$$

By supposing that on average in the ring wall the following relationship holds true

$$
\sigma=p t^{-1} R=p \tau^{-1}
$$

(3.6) takes the form

$$
E_{t}^{(i)}=E\left(\frac{\sigma_{y}^{(i) \beta}}{\sigma_{y}^{(i) \beta}+\beta\left(E \varepsilon_{y}^{(i)}-\sigma_{y}^{(i)}\right)\left(p \tau^{-1}\right)^{\beta-1}}\right) .
$$

According to the results from material testing on UOE pipes discussed in the previous section, with respect to the reference material of region (2) for which it is $\sigma_{y}^{(2)}=\sigma_{y}$, 
region (1) is considered to be characterised by an increased material strength and region (3) is considered to be characterised by a decreased material strength, that is,

$$
\sigma_{y}^{(1)}=(1+\alpha) \sigma_{y}, \quad \sigma_{y}^{(3)}=(1-\alpha) \sigma_{y}, \quad \alpha \in(0,1) \subset R
$$

The stress-strain curves for regions (1), (2), and (3) are shown in Figure 5. form

The general solution for each of the differential Equations (3.3) can be taken in the

$$
w^{(i)}(\theta)=A^{(i)} \sin k^{(i)} \theta+B^{(i)} \cos k^{(i)} \theta+C^{(i)} \cos n \theta, \quad i \equiv\{1,2,3\},
$$

with

$$
\begin{aligned}
k^{(i)} & =\sqrt{\left(1+\varphi^{(i)}\right)}, \\
C^{(i)} & =\frac{\varphi^{(i)} \omega R}{n^{2}-1-\varphi^{(i)}} .
\end{aligned}
$$

By virtue of (3.7), the axial force per unit length, $N$, in the ring wall is given by

$$
N^{(i)} \equiv N=\sigma t=p R, \quad \forall i \in\{1,2,3\},
$$

while the bending moment, $M^{(i)}$, at a generic section of the ring is given by

$$
M^{(i)}=p R \delta^{(i)}=p R\left[w^{(i)}(\theta)+\omega R \cos n \theta\right],
$$

where $\delta^{(i)}=w^{(i)}(\theta)+\omega R \cos n \theta$ represents the eccentricity of the axial force in the displaced configuration from the funicular curve of the external pressure.

The values of the unknown constants $A^{(i)}$ and $B^{(i)}$ in (3.10) can be determined by imposing the compatibility of slopes and displacements along the ring circumference. In fact, by virtue of the assumed symmetry about the $x$-axis, this is attained through the following set of boundary conditions:

$$
\begin{aligned}
\left.\left(w^{(1)}-w^{(2)}\right)\right|_{\theta=\theta_{c}} & =0,\left.\quad\left(w^{\prime(1)}-w^{\prime(2)}\right)\right|_{\theta=\theta_{c}}=0, \\
\left.\left(w^{(2)}-w^{(3)}\right)\right|_{\theta=\pi-\theta_{c}} & =0,\left.\quad\left(w^{\prime(2)}-w^{\prime(3)}\right)\right|_{\theta=\pi-\theta_{c}}=0, \\
\left.w^{\prime(1)}\right|_{\theta=0}=0, & \left.w^{\prime(3)}\right|_{\theta=\pi}=0 .
\end{aligned}
$$

Equations (3.15) represent a $(6 \times 6)$ algebraic system of linear equations that can be conveniently arranged in matrix form

$$
\mathbf{P U}=\mathbf{V}
$$




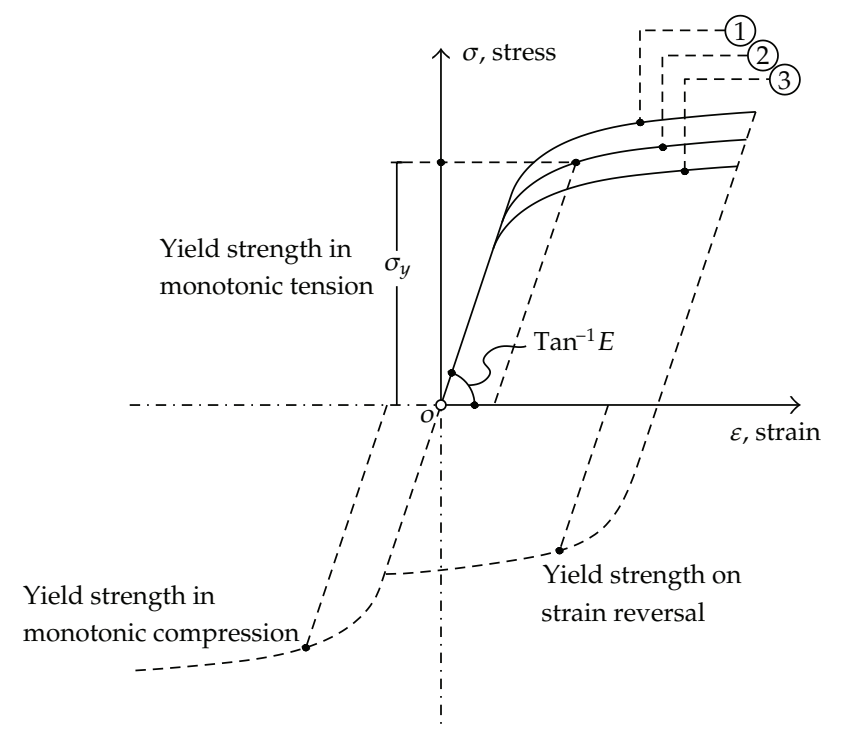

Figure 5: Stress-strain curves for the material regions (1) through (3).

where the vector $\mathbf{U}$ collects the six unknown coefficients $A^{(i)}$ and $B^{(i)}$ to be determined, that is,

$$
\mathbf{U}^{T}=\left\{U_{1}, U_{2}, U_{3}, U_{4}, U_{5}, U_{6}\right\} \equiv\left\{A^{(2)}, B^{(2)}, A^{(1)}, B^{(1)}, A^{(3)}, B^{(3)}\right\}
$$

and the entries of the vector $\mathbf{V}^{T}=\left\{V_{1}, V_{2}, V_{3}, V_{4}, V_{5}, V_{6}\right\}$ are given by

$$
\begin{gathered}
V_{1}=\left(C^{(1)}-C^{(2)}\right) \cos n \theta_{c}, \quad V_{2}=\left(C^{(2)}-C^{(1)}\right) n \sin n \theta_{c}, \\
V_{3}=\left(C^{(3)}-C^{(2)}\right) \cos n\left(\pi-\theta_{c}\right), \quad V_{4}=\left(C^{(2)}-C^{(3)}\right) n \sin n\left(\pi-\theta_{c}\right), \\
V_{5}=0, \quad V_{6}=-C^{(3)} n \sin n \pi .
\end{gathered}
$$

The nonzero entries $P_{i j}$ of the $(6 \times 6)$ matrix $\mathbf{P}$ in (3.16) can be explicitly written as follows:

$$
\begin{aligned}
& P_{11}=-\sin k^{(2)} \theta_{c}, \quad P_{12}=-\cos k^{(2)} \theta_{c}, \quad P_{13}=\sin k^{(1)} \theta_{c}, \quad P_{14}=\cos k^{(1)} \theta_{c}, \\
& P_{21}=-k^{(2)} \cos k^{(2)} \theta_{c}, \quad P_{22}=k^{(2)} \sin k^{(2)} \theta_{c}, \quad P_{23}=k^{(1)} \cos k^{(1)} \theta_{c,} \\
& P_{24}=-k^{(1)} \sin k^{(1)} \theta_{c}, \quad P_{31}=-\sin k^{(2)}\left(\pi-\theta_{c}\right), \quad P_{32}=-\cos k^{(2)}\left(\pi-\theta_{c}\right),
\end{aligned}
$$




$$
\begin{aligned}
& P_{35}=\sin k^{(3)}\left(\pi-\theta_{c}\right), \quad P_{36}=\cos k^{(3)}\left(\pi-\theta_{c}\right), \\
& P_{41}=-k^{(2)} \cos k^{(2)}\left(\pi-\theta_{c}\right), \quad P_{42}=k^{(2)} \sin k^{(2)}\left(\pi-\theta_{c}\right), \\
& P_{45}=k^{(3)} \cos k^{(3)}\left(\pi-\theta_{c}\right), \quad P_{46}=-k^{(3)} \sin k^{(3)}\left(\pi-\theta_{c}\right), \quad P_{53}=k^{(1)}, \\
& P_{65}=k^{(3)} \cos k^{(3)} \pi, \quad P_{66}=-k^{(3)} \sin k^{(3)} \pi,
\end{aligned}
$$

where the coefficients $k^{(i)}$ are given by (3.11).

Provided that det $\mathbf{P} \neq 0$, by means of Cramer's rule, the solution of (3.16) can be formally written as [15]

$$
\mathbf{U}=\mathbf{P}^{-1} \mathbf{V}=(\operatorname{det} \mathbf{P})^{-1} \tilde{\mathbf{P}} \mathbf{V}, \quad U_{i}=(\operatorname{det} \mathbf{P})^{-1} \sum_{j=1}^{6} \widetilde{P}_{j i} V_{j}
$$

where $\left\{\widetilde{\mathbf{P}}=\right.$ adj $\left.\mathbf{P},[\widetilde{\mathbf{P}}]_{i j} \equiv \widetilde{P}_{i j}\right\}$ represents the adjugate of the matrix $\mathbf{P}$. Substitution of (3.20) in (3.10) provides the displacement $w^{(i)}(\theta)$ for each ring sector as a function of the ring geometrical ratio, $\tau=t R^{-1}$, of the dimensionless amplitude of the imperfection, $\omega=w_{0} R^{-1}$, of the number of waves characterizing the initial imperfection shape of the ring, $n$, of the material Tangent modulus, $E_{t}^{(i)}$, of the amplitude of the region, $\theta_{c}$, and of the applied pressure, $p$.

Once the expression of the radial displacement, $w^{(i)}(\theta)$, is obtained, the expression of the bending moment $M^{(i)}(\theta)$ along the circumference can be evaluated from (3.14).

With reference to the sections of the ring wall where the maximum bending moment, $M_{\max }^{(i)}$, is achieved for each of the different material regions, the attainment of the ultimate carrying capacity at a certain section of the ring wall can be assumed to be ruled by the following well-known nonlinear yield equation [16]:

$$
\frac{M_{\max }}{M_{p}}+\left(\frac{N}{N_{p}}\right)^{2}-1=0,
$$

where

$$
N_{p}=\sigma_{y} t, \quad M_{p}=\frac{1}{4} \sigma_{y} t^{2} .
$$

However, when instability and plasticity phenomena coexist, the response of a cross-section of the ring is more realistically governed by an elastic-plastic behaviour. For this reason, reference is made to the following axial force-bending moment relationship:

$$
\frac{M_{\max }}{M_{p}}+\psi\left(\frac{N}{N_{p}}-1\right)=0, \quad \psi=\frac{1}{3}(2+\lambda),
$$


which covers a range of possible elastic-plastic axial force-bending moment interactions in the ring wall by means of a scalar parameter $\lambda \in[0,1]$, see Figure 6.

In fact, in the case $\lambda=0$ a condition of first local yielding is obtained, while $\lambda=1$ represents an intermediate elastic-plastic condition, characterized by the fact that in such a case the area of the admissible domain in the plane $n_{p}-m_{p}$, with $M / M_{P} \equiv m_{p}$ and $N / N_{P} \equiv$ $n_{p}$, constitutes the mean value of the areas of the admissible domains in the elastic $(\lambda=0)$ and fully plastic (3.21) limit conditions (see Figure 6), respectively. Without loss of generality, in the following (3.23) will be employed with $\lambda=1$, as widely accepted for a large number of problems involving carbon steel members [15].

Therefore, with reference to the sections of the ring wall where the maximum bending moment, $M_{\max }^{(i)}$, is achieved for each of the different material regions, the attainment of the ultimate carrying capacity at a certain section of the ring wall can be assumed to be ruled by the following axial force-bending moment relationship

$$
f^{(i)}=m_{p}^{(i)}+n_{p}^{(i)}-1=\frac{\left|M_{\max }^{(i)}\right|}{M_{p}^{(i)}}+\frac{N}{N_{p}^{(i)}}-1=0
$$

where $N$ is given by (3.13) and

$$
N_{p}^{(i)}=\sigma_{y}^{(i)} t, \quad M_{p}^{(i)}=\frac{1}{4} \sigma_{y}^{(i)} t^{2}
$$

It is worth noticing that, even if the analytical solutions for the displacements $w^{(i)}$ and the bending moments $M^{(i)}$ can be formally obtained by the solution of the problem, the location of the points at which the maximum bending moments are attained cannot be known a priori.

In fact, since the regions into which the circumference is divided are characterised by different material properties, it is

$$
M_{\max }^{(1)} \equiv \max _{0 \leq \theta \leq \theta_{c}}\left\{M^{(1)}(\theta)\right\}, \quad M_{\max }^{(2)} \equiv \max _{\theta_{c} \leq \theta \leq \pi-\theta_{c}}\left\{M^{(2)}(\theta)\right\}, \quad M_{\max }^{(3)} \equiv \max _{\pi-\theta_{c} \leq \theta \leq \pi}\left\{M^{(3)}(\theta)\right\} .
$$

Additionally, while in a homogeneous ring a number of fully yielded wall sections sufficient to activate a collapse mechanism always are concurrently born, regardless of the integer number of waves characterising the initial imperfection, $n$, for an inhomogeneous ring this is not generally the case. This means that, in principle, the occurrence of such fully yielded sections should be analysed for each region along the loading path. Clearly, such a procedure would render the proposed approach so lengthy to make it unpractical and to suggest, instead, always a recourse to numerical methods. 


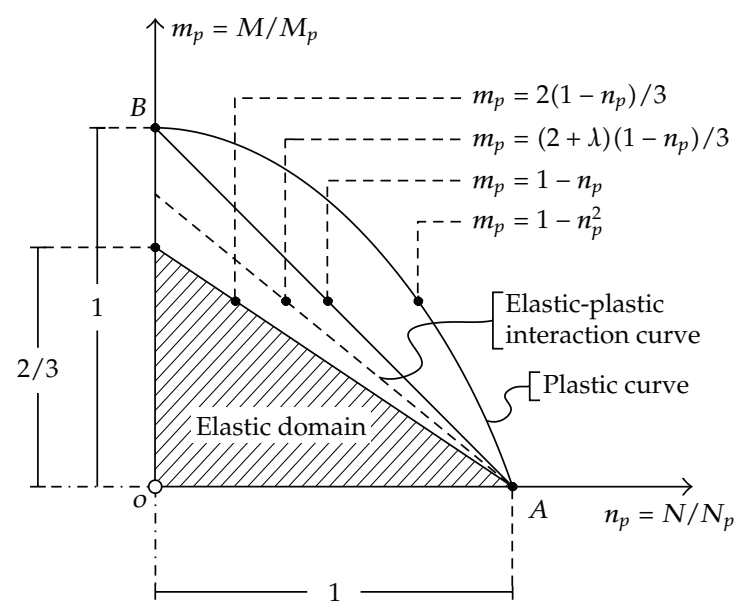

Figure 6: Elastic, elastic-plastic, and plastic loci in the $n_{p}-m_{p}$ plane.

However, the value of the external pressure corresponding to the first occurrence of at least one plastic hinge in the inhomogeneous ring can be directly obtained from (3.24) as the smallest of the roots of the following equations:

$$
\begin{aligned}
f^{(i)} & \equiv \frac{M_{\max }^{(i)}}{M_{p}^{(i)}}+\frac{N}{N_{p}^{(i)}}-1=f^{(i)}\left(\tau, \omega, n, E, \beta, \sigma_{y}^{(j)}, \varepsilon_{y}^{(j)}, p\right)=0, \\
& \Longrightarrow p_{c r}^{I} \equiv \min \left\{\min _{r_{i} \in N}\left[2 p_{r_{i}}^{(i)} \mid f^{(i)}=0\right]\right\},
\end{aligned}
$$

where $r_{i} \in N$ represents the number of roots of the equation $f^{(i)}=0$.

In the next Section it will be shown that this approach can provide a very accurate bound to the collapsing pressure of inhomogeneous rings.

Needless to say, in case all the regions share the same material properties, the presented development straightforwardly reduces to the result pertaining to a homogeneous ring, see [6].

\section{Case Studies and Assessment of Results}

The proposed analytical treatment has been assessed against a few case studies.

For all the examples the Ramberg Osgood material data for the region (2) (which corresponds to the $90^{\circ}-270^{\circ}$ positions), that is, the elasticity modulus, $E$, the yield strength, $\sigma_{\ell}$, and the corresponding elongation, $\varepsilon_{\ell}$, have been averaged by a number of experimental data sets provided by Tata Steel, with an example shown in Figure 7 . The exponent $n$, as usual, was obtained as a result of a standard error minimization procedure.

The yield strengths for the regions (1) and (3) (which, in turn, correspond to the $0^{\circ}$ and $180^{\circ}$ positions) were obtained by amplifying the yield strength of region (2) by a factor 1.1 and by reducing it by a factor 0.9 , respectively, according to (3.9). In this manner an increase 


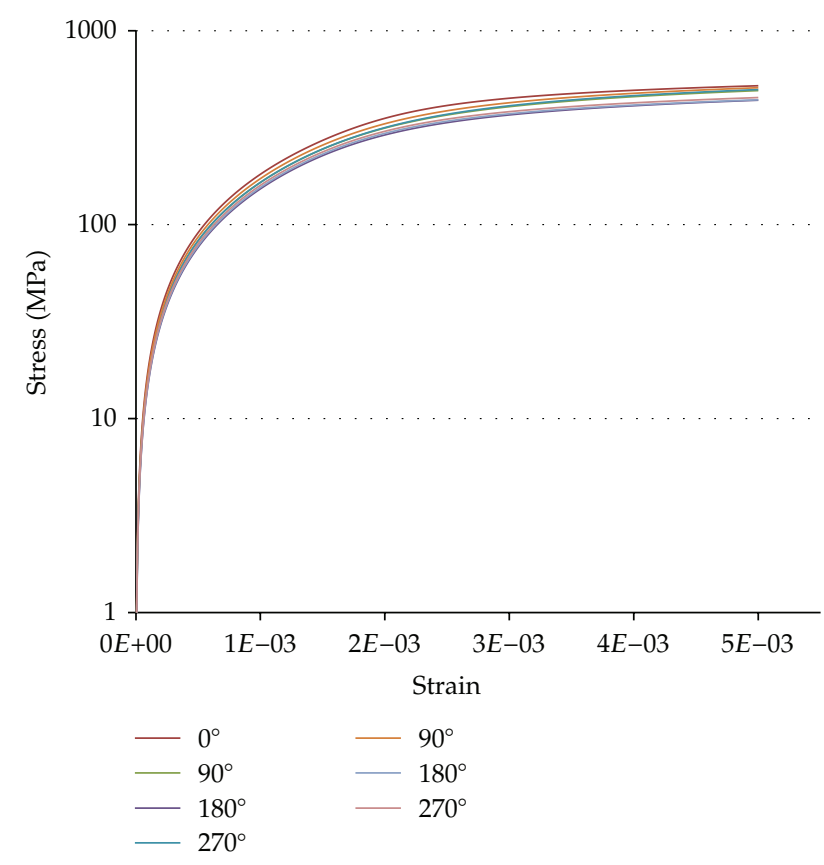

Figure 7: Some experimental stress-strain curves from samples taken along the pipe section (Tata, 2010).

in resistance was attributed to the weld region (1) at $0^{\circ}$ and a corresponding decrease in resistance was attributed to the region (3) at $180^{\circ}$.

The amplitude of the initial imperfection, $\omega$, was set equal to $0.001(\omega=1 / 1000)$ and the number of waves characterising the out of roundness, $n$, was set to 3 , which is the value experimentally assessed in [7] for the tested UOE formed pipes.

The case studies made reference to a ring characterised by a diameter of $457.2 \mathrm{~mm}$ and three different wall thicknesses, $t=32 \mathrm{~mm}, t=26 \mathrm{~mm}$, and $t=20 \mathrm{~mm}$, in order to deal with a range of $D /$ tspanning from moderately thick to relatively thin tubes. The amplitude of the four different material sectors, in absence of any precise measurement, was chosen with the purpose of magnifying the phenomenon, setting $\theta_{c}=\pi / 4$.

Figure 8 shows the contour plot of the yield function values, $f$, for a homogeneous (i.e., a ring uniformly characterised by the material properties of region (2) and an inhomogeneous ring at the first attainment of complete yielding of a section along the circumference $(t=26 \mathrm{~mm})$.

It is evident that in the case of the homogeneous ring a fully plastic status is achieved at the same time at four sections, located at $\theta=0^{\circ}, \theta=45^{\circ}, \theta=135^{\circ}$, and $\theta=180^{\circ}$, thus giving origin, on account of the symmetry about the $x$-axis, to a collapse mechanism. On the contrary, in the corresponding inhomogeneous ring, characterised by an increase of the material resistance in the region (1) $\left(\theta \in\left[0, \theta_{c}\right]\right)$ and by a corresponding decrease in the region (3) $\left(\theta \in\left[\pi-\theta_{c}, \pi\right]\right)$, a fully plastic status takes place at $\theta=180^{\circ}$ with all the remaining sections in the condition $\{(\theta, p) \mid f<0\}$ and no collapse taking place yet.

In order to validate the results, the case studies taken into consideration have been analysed by means of the commercial finite element (FE) package ANSYS [17]. 


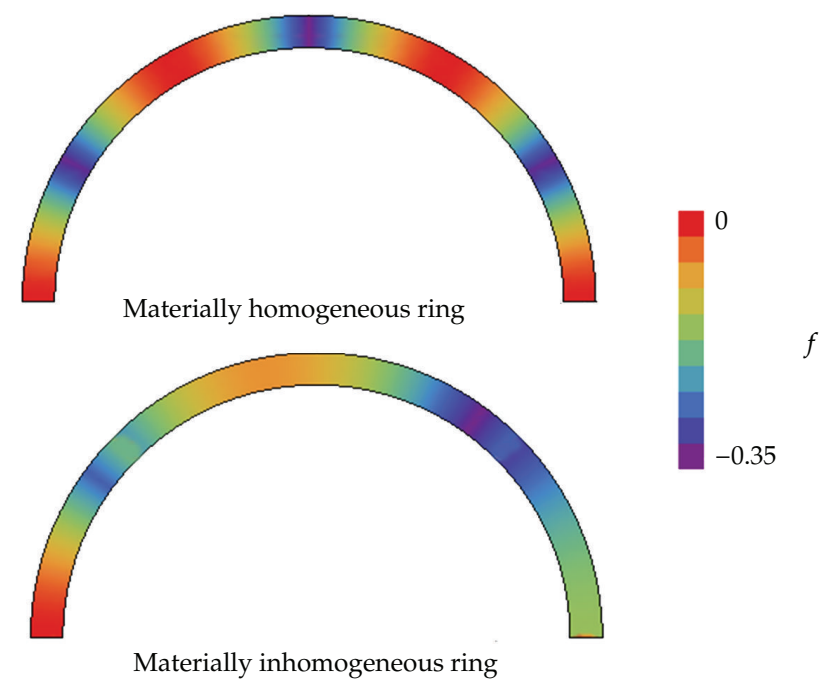

Figure 8: Contour plot of the yield function values, $f$, for the homogeneous and the inhomogeneous cases at the complete yielding of the first section along the circumference $(t=26 \mathrm{~mm}, D=457.2 \mathrm{~mm}, \omega=1 / 1000$, $n=3$, and $\left.\theta_{c}=\pi / 4\right)$.

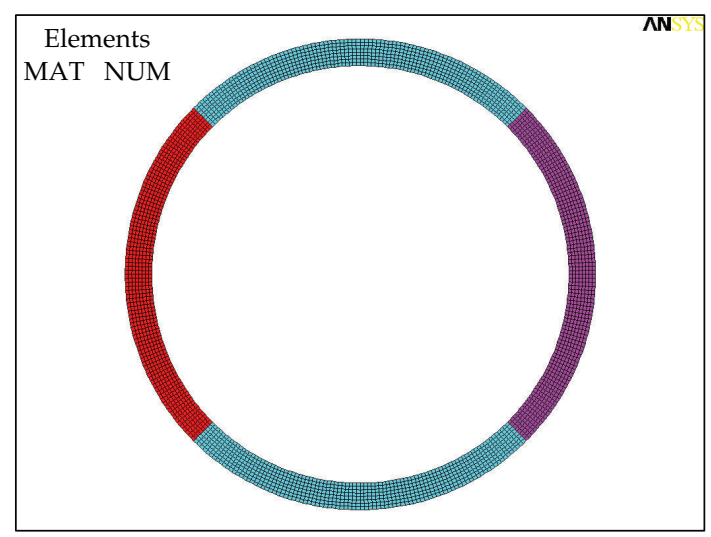

Figure 9: FE modelling of a inhomogeneous ring.

The model was carefully calibrated against carefully conducted experimental results [7] and every ring was modelled by means of 2880 PLANE182-2D 4 Nodes elements in plane stress, as shown in Figure 9.

Table 1 collects the results from the analytical and FE analyses.

It is immediate to notice that the analytical and the FE results agree very well for all the case studies. Moreover, it can be pointed out that the lower bounds from the proposed treatment essentially coincide with the collapse pressures from the FE analyses. In fact, with reference to Figure 10, which shows the maps of the evolving hoop strains at impending collapse for a progressive shortening of the horizontal diameter of about $1 \%(t=26 \mathrm{~mm})$, it appears evident that the plastic strains very swiftly evolve from the condition on the left, which basically corresponds to the complete yielding of the first section along the circumference shown in Figure 8, to the collapsing mechanism on the right. Thus, 
Table 1: Collapse pressures $[\mathrm{MPa}]$ for homogeneous rings $(\mathrm{H})$ and lower bound pressures $[\mathrm{MPa}]$ for inhomogeneous rings (I) $\left(D=457.2 \mathrm{~mm}, \omega=1 / 1000, n=3\right.$, and $\left.\theta_{c}=\pi / 4\right)$.

\begin{tabular}{|c|c|c|c|c|c|c|c|c|c|c|c|}
\hline \multicolumn{4}{|c|}{$t=20 \mathrm{~mm}$} & \multicolumn{4}{|c|}{$t=26 \mathrm{~mm}$} & \multicolumn{4}{|c|}{$t=32 \mathrm{~mm}$} \\
\hline \multicolumn{2}{|c|}{$\mathrm{H}$} & \multicolumn{2}{|c|}{$\mathrm{I}$} & \multicolumn{2}{|c|}{$\mathrm{H}$} & \multicolumn{2}{|c|}{ I } & \multicolumn{2}{|c|}{$\mathrm{H}$} & \multicolumn{2}{|c|}{ I } \\
\hline Theory & FEM & Theory & FEM & Theory & FEM & Theory & FEM & Theory & FEM & Theory & FEM \\
\hline 35.27 & 34.59 & 27.88 & 28.07 & 51.20 & 50.83 & 42.83 & 42.34 & 68.44 & 68.43 & 58.56 & 57.60 \\
\hline
\end{tabular}

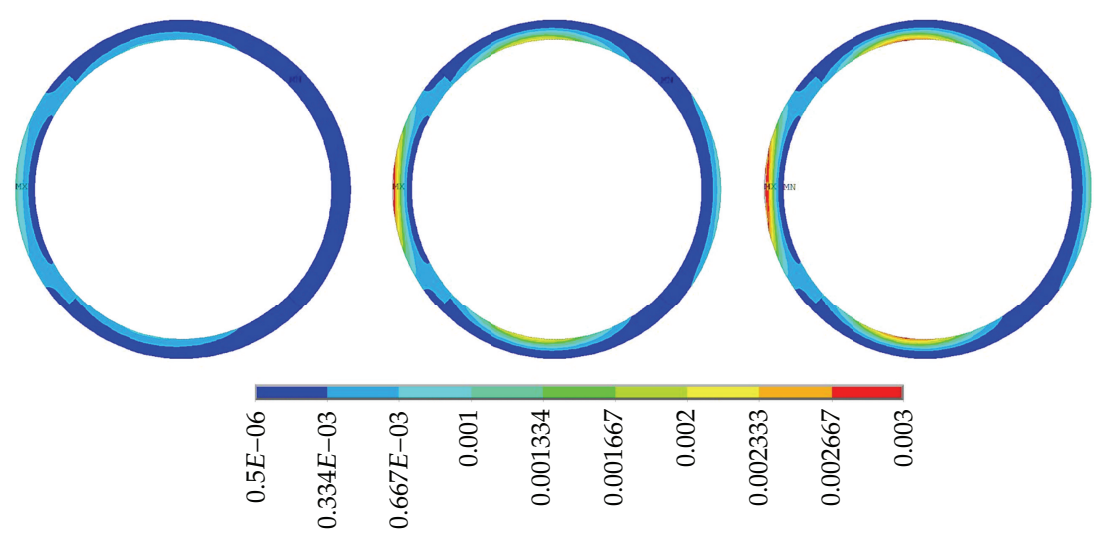

Figure 10: Evolution of the hoop strains at impending collapse $\left(p_{\mathrm{cr}}=42.34 \mathrm{MPa}\right)$ from the FE analysis of a inhomogeneous ring $\left(t=26 \mathrm{~mm}, D=457.2 \mathrm{~mm}, \omega=1 / 1000, n=3\right.$, and $\left.\theta_{c}=\pi / 4\right)$.

the attainment of the complete yielding of the first section along the circumference practically marks the carrying capacity of the ring.

Overall, it can be stated that the attainment of a fully plastic state takes place in the inhomogeneous ring at a value of the external pressure which ranges from about 81 to $85 \%$ of the value of the critical pressure for the homogeneous case. Therefore, the reduction in the lower bound to the collapsing pressure results in being higher in percentage than the variation in the material properties. Also, the decrease in the carrying capacity with respect to the homogeneous case results in being directly proportional to the $D / t$ ratio, as it can be seen from Table 1.

A preliminary confirmation of the results for the case $t=32 \mathrm{~mm}$ has also been obtained experimentally, with a value for the critical pressure of about $55.40 \mathrm{MPa}$.

\section{Conclusions}

In the present work an analytical approach that can provide an adequate prediction of the collapse pressure of a pipe section in the case of UOE formed deep-water pipelines and that accounts for the effects of geometrical data and varying material properties has been presented and discussed. The proposed approach has its route in the fundamental mechanics of the problem and is capable of blending transitions between elastic collapse and plastic collapse.

With respect to the recourse to numerical methods, such as finite element analyses, it is felt that the proposed approach can provide a thorough understanding of the actual mechanisms involved in the buckling of the pipe and, by focusing on the few parameters 
which govern the phenomenon, may open the way to improvements in performance through optimisation of the pipe manufacturing process.

\section{Acknowledgments}

The present study has been motivated and financially supported by Tata Steel, UK. In particular, the authors are grateful to Dr. Richard Freeman for all his assistance.

\section{References}

[1] F. Guarracino, M. Fraldi, and A. Giordano, "Analysis of testing methods of pipelines for limit state design," Applied Ocean Research, vol. 30, no. 4, pp. 297-304, 2008.

[2] F. Guarracino, A. C. Walker, and A. Giordano, "Effects of boundary conditions on testing of pipes and finite element modelling," International Journal of Pressure Vessels and Piping, vol. 86, no. 2-3, pp. 196-206, 2009.

[3] F. Guarracino, "A simple formula for complementing FE analyses in the estimation of the effects of local conditions in circular cylindrical shells," Computer Modeling in Engineering and Sciences, vol. 72, no. 3, pp. 167-184, 2011.

[4] ASTM Standard E9, Standard Test Methods of Compression Testing of Metallic Materials at Room Temperature, ASTM International, West Conshohocken, PA, USA, 2009.

[5] M. S. Hoo Fatt, "Elastic-plastic collapse of non-uniform cylindrical shells subjected to uniform external pressure," Thin-Walled Structures, vol. 35, no. 2, pp. 117-137, 1999.

[6] M. Fraldi and F. Guarracino, "An improved formulation for the assessment of the capacity load of circular rings and cylindrical shells under external pressure. Part 1. Analytical derivation," ThinWalled Structures, vol. 49, no. 9, pp. 1054-1061, 2011.

[7] M. Fraldi, R. Freeman, S. Slater, A. C. Walker, and F. Guarracino, "An improved formulation for the assessment of the capacity load of circular rings and cylindrical shells under external pressure. Part 2. A comparative study with design codes prescriptions, experimental results and numerical simulations," Thin-Walled Structures, vol. 49, no. 9, pp. 1062-1070, 2011.

[8] F. Guarracino, M. Fraldi, S. Slater, and R. Freeman, "Hydrostatic collapse of deepwater pipelines: a rigorous analytical approach," in Proceedings of the Offshore Technology Conference, Houston, Tex, USA, May 2011.

[9] S. P. Timoshenko, Theory of Elastic Stability, McGraw-Hill Book, New York, NY, USA, 1961.

[10] L. H. Donnell, "Effect of imperfections on buckling of thin cylinders under external pressure," Journal of Applied Mechanics, vol. 23, pp. 569-575, 1956.

[11] G. Gerard, "Plastic stability theory of thin shells," Journal of the Aeronautical Sciences, vol. 24, pp. 269274, 1957.

[12] F. Guarracino, "Considerations on the numerical analysis of initial post-buckling behaviour in plates and beams," Thin-Walled Structures, vol. 45, no. 10-11, pp. 845-848, 2007.

[13] G. Gerard, Introduction to Structural Stability Theory, McGraw-Hill, 1962.

[14] W. Ramberg and W. R. Osgood, "Description of stress-strain curves by three parameters," Technical Note 902, NACA, 1943.

[15] N. Jacobson, Basic Algebra, vol. 1, Dover, 2009.

[16] M. Zyczkowski, Combined Loadings in Theory of Plasticity, Polish Scientific, Warsaw, Poland, 1981.

[17] ANSYS 13.0 User's Documentation, ANSYS, Canonburg, Pa, USA, 2011. 


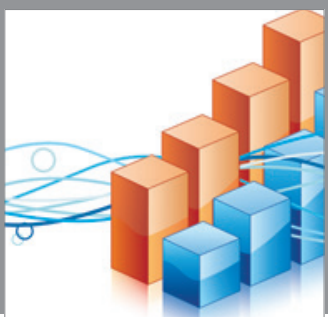

Advances in

Operations Research

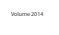

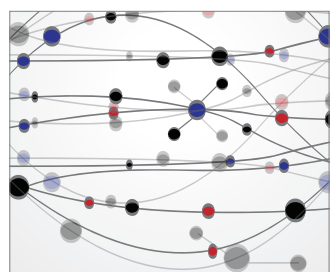

\section{The Scientific} World Journal
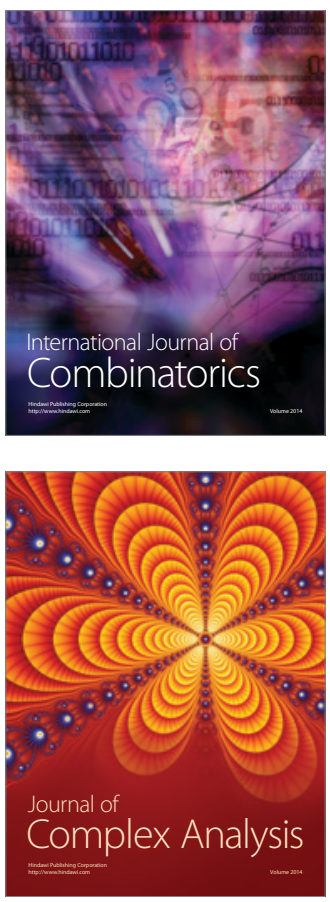

International Journal of

Mathematics and

Mathematical

Sciences
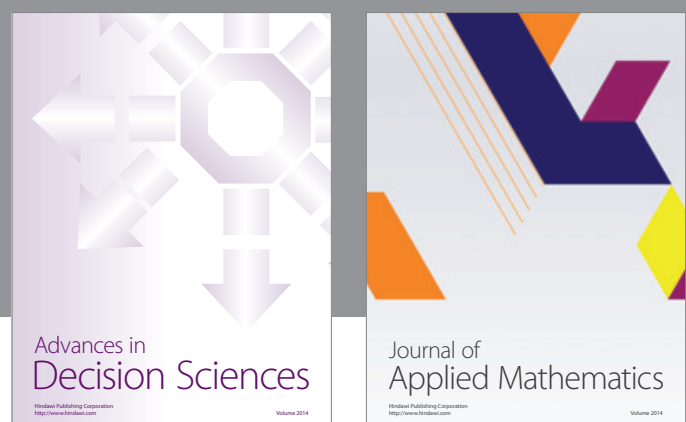

Journal of

Applied Mathematics
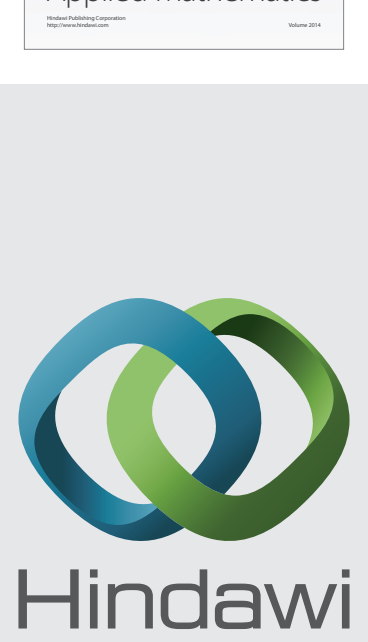

Submit your manuscripts at http://www.hindawi.com
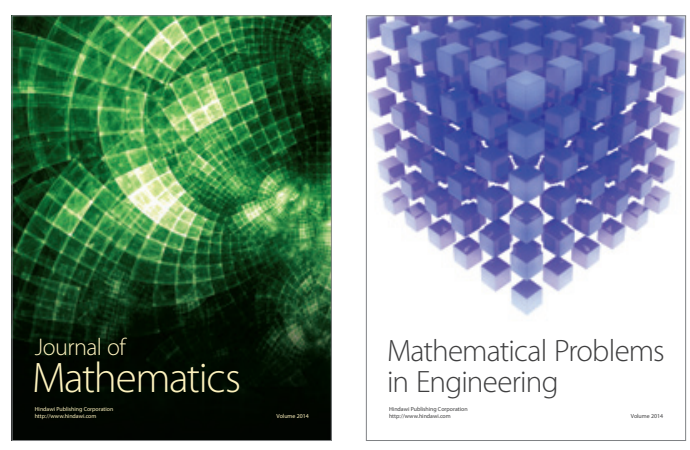

Mathematical Problems in Engineering
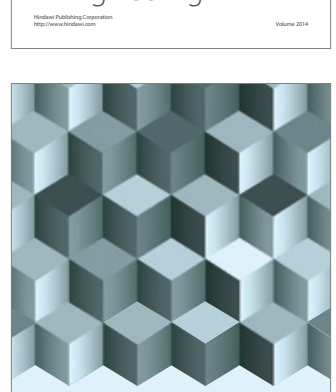

Journal of

Function Spaces
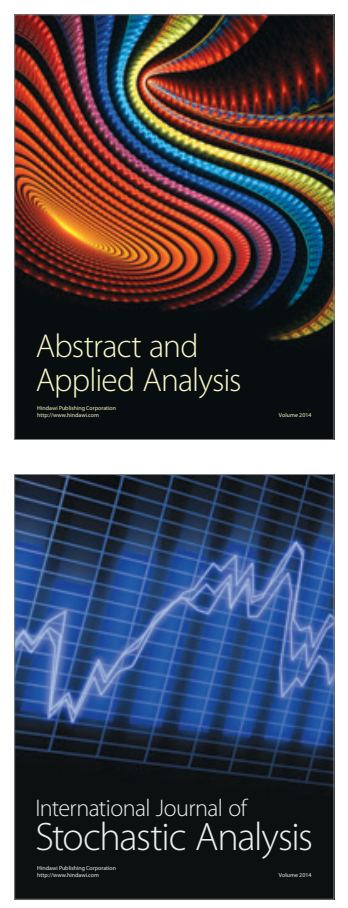

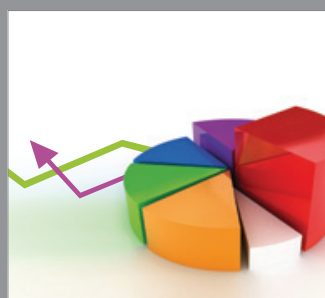

ournal of

Probability and Statistics

Promensencen
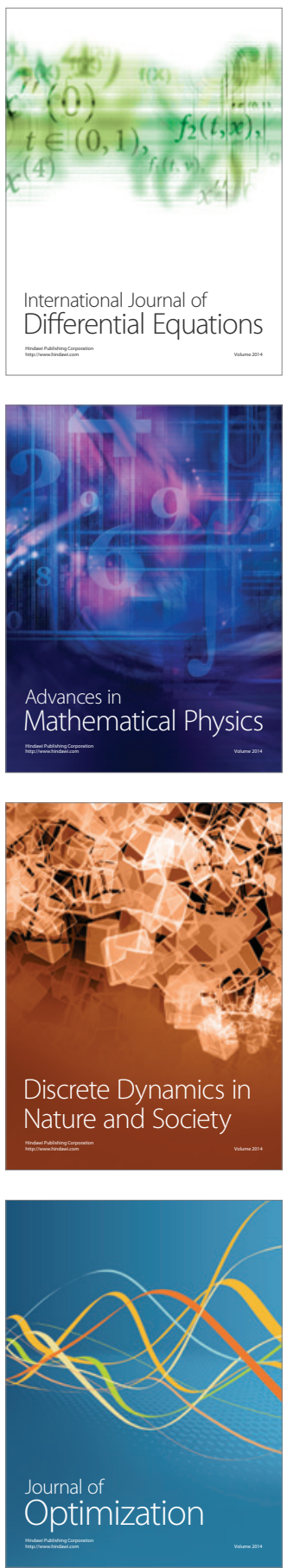